\title{
Endovascular Stroke Therapy Results Improve over Time: The 'Learning Curve' at a New Comprehensive Stoke Center
}

\author{
Ethan A. Benardete Anil K. Nair \\ Clinical Neuroscience Program, Kennedy Memorial Hospital, Washington Township, N.J., and \\ Department of Neurological Surgery, Thomas Jefferson University, Philadelphia, Pa., USA
}

\section{Key Words}

Stroke · Stroke center · Endovascular stroke therapy · Stroke care quality · Quality improvement $\cdot$ Stroke care organization

\begin{abstract}
Background: The requirements for a comprehensive stroke center (CSC) include the capability to perform endovascular stroke therapy (EST). EST is a complex process requiring early identification of appropriate patients and effective delivery of intervention. In order to provide prompt intervention for stroke, CSCs have been established away from large academic centers in community-based hospitals. We hypothesized that quantifiable improvements would occur during the first 2 years of a community-based CSC as the processes and personnel evolved. We report the results over time of EST at a new community-based CSC. Methods: We have retrospectively analyzed demographic data and outcome metrics of EST from the initiation phase of a new community-based CSC. Data was divided into year 1 and year 2. Statistical analysis (Student's $t$ test and Fisher's exact test) was performed to compare the patient population and outcomes across the two time periods. Outcome variables included the thrombolysis in cerebral infarction (TICI) score, a change in the NIH stroke scale score and the modified Rankin Scale (mRS) score. Analysis of variance (ANOVA) was used to statistically analyze the relationship between population variables and outcome. Computed tomography (CT) angiography and CT perfusion analysis were used to select patients for EST. Approximately half of the patients undergoing EST were excluded from receiving intravenous recombinant tissue plasminogen activator (IV rt-PA) by standard criteria, while the other half showed no sign of improvement following $1 \mathrm{~h}$ of IV rt-PA treatment. Mechanical thrombolysis with a stentriever was performed in the majority of cases with or without intra-arterial medication. The majority of treated occlusions were in the middle cerebral artery. Results: A total of 18 patients underwent EST during year 1 and year 2. A statistically significant increase in good
\end{abstract}


outcomes (mRS score $\leq 2$ at discharge) was seen from year 1 to year $2(p=0.05)$. There were trends towards faster interventions, decreased complications and mortality as well as an improved TICI score from year 1 to year 2 . With ANOVA, mortality was statistically correlated with age $(p=0.06)$, while decreases in the NIH stroke scale (NIHSS) score following EST correlated with decreased mortality $(p=0.01)$. A higher TICI score was significantly associated with a decreased NIHSS score following EST $(p=0.01)$. Conclusions: At a new communitybased CSC, improved outcome occurred from year 1 to year 2, and trends towards decreased mortality, fewer complications, and improved revascularization were observed. Furthermore, the data shows that improvement in NIHSS score after EST is associated with decreased mortality following stroke in this setting, implying a net benefit.

(C) 2015 S. Karger AG, Basel

\section{Introduction}

The Brain Attack Coalition (BAC) defined the requirements for the establishment of primary stroke centers in 2000 and comprehensive stroke centers (CSCs) in 2005 [1, 2]. Subsequently, many hospital systems within the United States have established CSCs. In late 2011, the Kennedy Health System (Camden and Gloucester Counties, N.J., USA) initiated a CSC at one of its three campuses. This CSC is a referral center for all three hospitals within its health system as well as for the local community. This CSC is also a joint venture with a regional academic medical center (Thomas Jefferson University, Philadelphia, Pa., USA). Neurologists employed by the hospital and telemedicine stroke neurologists based at the academic center provide vascular neurology coverage. Endovascular stroke therapy (EST) is carried out by two interventional neurosurgeons with joint appointments at both the hospital system and the academic center. The purpose of this article is to review the outcomes of patients who received EST at a new CSC and look at trends in outcomes over time. In the literature, we could not identify any normative data on the initiation phase of a CSC in the United States. Our contribution is to help define expected progress during such a start-up phase and the utilization of EST during that period.

Our hypothesis was that over time, outcomes improve as the system for providing EST evolves in terms of personnel, equipment, and protocols. Our data showed that there was a statistically significant increase in good outcomes from year 1 to year 2 . There were also trends towards decreased mortality, decreased complications, and increased revascularization over time. Furthermore, improved revascularization and decreased NIH stroke scale (NIHSS) scores were significantly associated with decreased mortality and improved outcomes.

\section{Methods}

A retrospective chart review was conducted on patients admitted to the CSC with the diagnosis of acute ischemic stroke and treated with EST from January 2012 (start of the CSC) through December 2013. Demographic data was collected along with outcome and treatment data including method of revascularization, thrombolysis in cerebral infarction (TICI) score $[3,4]$, and NIHSS score as established by the National Institute of Neurological Disorders and Stroke (NINDS). Data is divided into year 1 (January to December 2012) and year 2 (January to December 2013). For parametric data, significance testing was performed with a two-tailed Student $\mathrm{t}$ test, assuming equal variances using Excel 2011 (Microsoft, Inc., Redmond, Wash., USA). For nonparametric data, Fisher's exact test for $2 \times 2$ contingency tables and one-way analysis of variance (ANOVA) testing was performed with SPSS (IBM, Inc., Armonk, N.Y., USA). 


\section{Demographic Data}

For each patient, several stroke risk factors were recorded including age, history of prior stroke, hypertension, hyperlipidemia, atrial fibrillation, and smoking. Hypertension was defined as a previous history of hypertension as reported by the patient or family or an admission systolic blood pressure $>180 \mathrm{~mm} \mathrm{Hg}$. Hyperlipidemia was recorded if the patient reported a history of hyperlipidemia or had total cholesterol $>200 \mathrm{mg} / \mathrm{dl}$ on initial laboratory screening. Atrial fibrillation was recorded if the patient or family reported a history of atrial fibrillation or electrocardiography documented atrial fibrillation on admission.

\section{Selection for EST}

The selection process for EST is described below; the process was not altered during the entire study period. Acute stroke patients were initially evaluated in the emergency department (ED) of one of the three hospitals in the system by an ED physician. A noncontrast head computed tomography (CT) scan was obtained, and a neurology consultation was requested. If the in-house neurologist was unavailable, a university-based neurologist conducted a telemedicine stroke consult. Standard criteria for administering intravenous recombinant tissue plasminogen activator (IV rt-PA) (Activase; Genentech, South San Francisco, Calif., USA) were used [5]. If the patient was ineligible for IV rt-PA administration based on standard criteria or failed to improve after $1 \mathrm{~h}$ of IV rt-PA administration, a CT perfusion scan of the brain and a CT angiogram of the brain and the neck were performed. In multiple studies, CT perfusion of the brain has been noted to be valuable in defining penumbra versus core infarct and avoiding posttreatment hemorrhage [6]. If, in those studies, a major perfusion deficit without an accompanying area of decreased blood volume (ischemic penumbra) was identified, a review of the CT angiogram was undertaken to identify a corresponding occluded vessel [7]. If such a vessel was identified, the patient was deemed a candidate for EST unless the decreased blood volume on perfusion imaging exceeded one third of the corresponding middle cerebral artery (MCA) territory. For posterior circulation ischemia, the decision to proceed with EST was based on whether there was major vessel occlusion on CT angiography, major neurological deficit, and significant penumbra on CT perfusion. Door-to-needle (DTN) time was the time from ED arrival to delivery of IV rt-PA for the patients who were able to receive the medication. Door-to-intervention (DTI) time was the time from ED arrival to procedure start time in the interventional suite. Procedure length was the time from procedure start to recorded procedure end time (catheters and sheaths removed from patient).

\section{Interventional Methods and Outcomes}

General endotracheal anesthesia was used for all interventions. Electroencephalography, somatosensory evoked potentials, and brainstem auditory evoked responses were recorded during the procedures. Arterial access was obtained through the common femoral artery. A large bore sheath (7 or $8 \mathrm{Fr}$ ) was placed. Diagnostic angiography was first performed to confirm an arterial occlusion. Next, a guide catheter was brought up close to the skull base or into the petrous carotid. Depending on the operator's preference, either thromboaspiration or mechanical retrieval (stentriever) was used. Thromboaspiration was performed with the Penumbra system (Penumbra Inc., Alameda, Calif., USA) using the standard technique. Mechanical retrieval based on a stentriever was done with either the Solitaire FR (ev3 Neurovascular, Irvine, Calif., USA) or the Trevo Pro (Stryker Neurovascular, Fremont, Calif., USA). In two cases, emergent carotid angioplasty and stenting were performed following EST; a Viatrac balloon, Rx Acculink carotid stent and Emboshield NAV6 embolic protection device were used (Abbott Laboratories, Abbott Park, Ill., USA). At the operator's discretion, small doses (2-5 mg) of either rt-PA and/or abciximab (2-5 mg) (Reopro; Eli Lilly and Co., Indianapolis, Ind., USA) were administered intra-arterially through the catheter during some EST procedures. In no case was intra-arterial (IA) medication the sole treatment. 


\section{Cerebirovascular \\ Diseases}

Table 1. Demographic data for acute stroke patients treated with EST in year 1 and year 2 at a new CSC

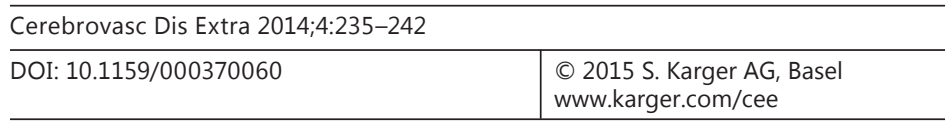

Benardete and Nair: Endovascular Stroke Therapy Results Improve over Time: The 'Learning Curve' at a New Comprehensive Stoke Center

\begin{tabular}{llll}
\hline & Year 1 & Year 2 & $\mathrm{p}$ \\
\hline Mean age, years & 78.4 & 66.1 & 0.03 \\
Min.-max. & $63-89$ & $34-88$ & \\
Mean NIHSS score & 19 & 14 & 0.16 \\
Min.-max. & $8-25$ & $6-21$ & \\
Male/female & $3 / 8$ & $4 / 3$ & 0.33 \\
\% prior stroke & $0(0 / 11)$ & $14(1 / 7)$ & 0.39 \\
\% smoker & $9(1 / 11)$ & $29(2 / 7)$ & 0.53 \\
\% HL & $64(7 / 11)$ & $85(6 / 7)$ & 0.60 \\
\% Afib & $45(5 / 11)$ & $28(2 / 7)$ & 0.64 \\
\% HTN & $82(9 / 11)$ & $71(5 / 7)$ & 1.00 \\
\% IV rt-PA & $55(6 / 11)$ & $57(4 / 7)$ & 1.00 \\
\hline
\end{tabular}

Mean NIHSS score $=$ Mean NIHSS score on admission; Min. - max. = minimum to maximum values; \% Afib = percentage of patients with atrial fibrillation; \% HL = percentage of patients with hyperlipidemia; $\%$ HTN = percentage of patients with hypertension; \% IV rt-PA = percentage of patients who had received IV rt-PA prior to ET. $p$ values are based on Fisher's exact test (see Methods section).

NIHSS scores were calculated on admission and $24 \mathrm{~h}$ after treatment. The degree of revascularization was assessed using the TICI scale as originally described by Higashida et al. [4]. Disability at the time of discharge was measured based on the modified Rankin Scale (mRS) [8].

\section{Results}

\section{Demographic Data}

The entire Kennedy Memorial Hospital system receives approximately 145,000 emergency room visits per year. Hospital records indicate that during the 2-year period, 1,644 patients were admitted to the hospital system with a diagnosis of stroke or transient ischemic attack. Of these, $91(5.5 \%)$ received IV rt-PA. Nineteen patients (1.1\%) were identified for EST treatment during the analysis period; however, 1 patient was transferred to the academic center when the biplane fluoroscopy equipment malfunctioned at the beginning of the procedure and could not be immediately repaired. This patient was excluded from the analysis.

The average age of the patient population was 74.6 years. During the first year, the average age was 78.3, and 66.1 years in the second year. This difference was statistically significant although selection criteria did not change from year 1 to year 2 . In total, 7 male and 11 female patients were treated. The mean NIHSS score prior to intervention in year 1 was 19 , and in year 2 , it was $14(\mathrm{p}=0.16)$. Hypertension, hyperlipidemia, and atrial fibrillation were highly represented among the patient population, while smoking and history of prior stroke were not (table 1).

In both year 1 and year 2, approximately half of the patients received IV rt-PA prior to intervention (table 1). In order to be candidates for EST, these patients continued to have a significant deficit after $1 \mathrm{~h}$ of IV rt-PA administration. CT perfusion and CT angiography were also required to demonstrate an ischemic penumbra and a vessel occlusion. Other reasons for referral for EST in both year 1 and year 2 included: age $>80$ years ( 2 patients), unknown time of onset of symptoms ('wake-up strokes') ( 5 patients), $>4.5 \mathrm{~h}$ from symptom onset ( 1 patient), and recent gastrointestinal hemorrhage (1 patient). For both year 1 and year 2 , the majority 


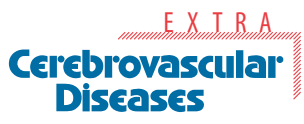

Table 2. Comparison of outcome and procedure data on acute stroke patients treated with EST at a new CSC in year 1 and year 2 (Fisher's exact test)
Table 3. Comparison of procedure and outcome variables from year 1 and year 2 at a new CSC

\begin{tabular}{l|l}
\hline \multicolumn{2}{l}{ Cerebrovasc Dis Extra 2014;4:235-242 } \\
\hline DOI: $10.1159 / 000370060$ & $\begin{array}{l}\text { @ 2015 S. Karger AG, Basel } \\
\text { www.karger.com/cee }\end{array}$ \\
\hline
\end{tabular}

Benardete and Nair: Endovascular Stroke Therapy Results Improve over Time: The 'Learning Curve' at a New Comprehensive Stoke Center

\begin{tabular}{lcrl}
\hline & Year 1 & Year 2 & $\mathrm{p}$ \\
\hline \% discharge mRS score $\leq 2$ & $9(1 / 11)$ & $57(4 / 7)$ & 0.05 \\
\%TICI score 3 & $27(3 / 11)$ & $57(4 / 7)$ & 0.33 \\
\% 30-day mortality & $36(4 / 11)$ & $14(1 / 7)$ & 0.60 \\
\% MCA & $90(10 / 11)$ & $100(7 / 7)$ & 1.00 \\
\% complication & $27(3 / 11)$ & $14(1 / 7)$ & 1.00 \\
\% stentriever & $73(8 / 11)$ & $85(6 / 7)$ & 1.00 \\
\% TICI score $\geq 2 \mathrm{~b}$ & $64(7 / 11)$ & $71(5 / 7)$ & 1.00 \\
\% IA medications & $36(4 / 11)$ & $43(3 / 7)$ & 1.00 \\
\hline
\end{tabular}

The fraction in parentheses is the number of patients with characteristics over the total. $\%$ discharge $\mathrm{mRS}$ score $\leq 2=$ percentage of patients at discharge who had an mRS score $\leq 2$ (independent); $\%$ TICI score $3=$ percentage of patients with complete revascularization on the TICI scale; $\%$ MCA = percentage of patients with an MCA occlusion on angiography; \% complication $=$ percentage of patients who had a procedural complication; $\%$ stentreiver $=$ percentage of patients treated with a stentriever as the primary endovascular device; $\%$ TICI $\geq 2 \mathrm{~b}$ score = percentage of patients with a revascularization score on the TICI scale of $2 \mathrm{~b}$ or higher; \% IA medications $=$ percentage of patients that received IA rt-PA or abciximab as an adjunct to their procedure. $\mathrm{p}$ values are based on Fisher's exact test.

\begin{tabular}{lccc}
\hline & Year 1 & Year 2 & $\mathrm{p}$ \\
\hline Mean DTN, min & 57.5 & 36.3 & 0.03 \\
Min.-max. & $54-121$ & $35-37$ & \\
Mean discharge mRS score & 4.4 & 2.8 & 0.18 \\
Min.-max. & $2-6$ & $1-6$ & \\
Mean DTI, min & 157 & 136 & 0.40 \\
Min.-max. & $70-222$ & $72-201$ & \\
Mean procedure length, min & 135 & 117 & 0.51 \\
Min.-max. & $49-234$ & $50-217$ & \\
Mean $\Delta$ NIHSS score & 5 & 6 & 0.88 \\
$\Delta$ NIHSS range & $(-13)-21$ & $0-14$ & \\
\hline
\end{tabular}

Mean $\Delta$ NIHSS score $=$ Average decrease in NIHSS following the procedure at $24 \mathrm{~h}$ after the procedure. The $\mathrm{p}$ value is based on significance testing as described in the text. Min. - max. $=$ minimum to maximum values.

of patients had MCA occlusions (year 1,90\%; year 2,100\%). The proportion of left ('dominant') hemisphere occlusions was not significantly different between year 1 and year 2 ( 0.7 vs. 0.43 , $\mathrm{p}=0.35$ ).

\section{Outcome Data}

The proportion of patients receiving EST with a good outcome (defined as mRS at discharge $\leq 2$ ) was significantly higher in year 2 compared to year $1(p=0.05$; table 2 ). There were also trends towards other outcome improvements in year 2 compared to year 1, but these did not reach statistical significance (table 2). For example, the 30-day mortality rate fell from 36 to $14 \%$ from year 1 to year $2(p=0.6)$, while the rate of complete revascularization (TICI score $=3$ ) improved from year 1 to year 2 (absolute increase $30 \%, p=0.33$ ). The mean improvement in the NIHSS score $24 \mathrm{~h}$ after the procedure was similar over the two periods (year 1 , score 5 ; year 2 , score 6) (table 3). 


\section{Cerebirovascular \\ Diseases}

Table 4. Results of the one-way ANOVA on pooled data from year 1 and year 2

\begin{tabular}{|c|c|c|}
\hline \multicolumn{3}{|c|}{ Cerebrovasc Dis Extra 2014;4:235-242 } \\
\hline DOI: $10.1159 / 000370060$ & \multicolumn{2}{|c|}{$\begin{array}{l}\text { (C) } 2015 \text { S. Karger AG, Basel } \\
\text { www.karger.com/cee }\end{array}$} \\
\hline \multicolumn{3}{|c|}{$\begin{array}{l}\text { Benardete and Nair: Endovascular Stroke Therapy Results Improve over Time: The } \\
\text { 'Learning Curve' at a New Comprehensive Stoke Center }\end{array}$} \\
\hline Variable 1 & Variable 2 & $\mathrm{p}$ \\
\hline$\Delta$ NIHSS score & 30-day mortality & 0.01 \\
\hline Age & 30-day mortality & 0.06 \\
\hline DTI time & 30-day mortality & 0.09 \\
\hline Procedure length & 30-day mortality & 0.12 \\
\hline Preoperative NIHSS score & 30-day mortality & 0.50 \\
\hline$\Delta$ NIHSS score & TICI score $\geq 2 b$ & 0.01 \\
\hline DTI time & TICI score $\geq 2 \mathrm{~b}$ & 0.14 \\
\hline Procedure length & TICI score $\geq 2 \mathrm{~b}$ & 0.32 \\
\hline Age & TICI score $\geq 2 \mathrm{~b}$ & 0.72 \\
\hline Preoperative NIHSS score & TICI score $\geq 2 \mathrm{~b}$ & 0.76 \\
\hline
\end{tabular}

$\Delta$ NIHSS score $=$ Change in NIHSS score $24 \mathrm{~h}$ following the procedure . 30 -day mortality and TICI score $\geq 2 \mathrm{~b}$ are categorical variables.

With data from both year 1 and year 2, one-way ANOVA testing was performed to analyze the relationship between several variables (age, DTI time, NIHSS score change, and procedure length) and 30-day mortality (table 4). A similar analysis was done between those variables and the TICI score (TICI score $\geq 2 \mathrm{~b}$ ) (table 4). Notably, NIHSS score improvement was significantly associated with decreased 30 -day mortality $(p=0.008)$. We found a statistically significant trend between 30-day mortality and increasing age $(p=0.06)$ as well as between DTI time and mortality $(\mathrm{p}=0.09)$. The other variables were not significantly associated with 30 -day mortality. A TICI score $\geq 2 \mathrm{~b}$ was significantly associated with improvement in NIHSS score $(p=0.01)$. This result is similar to recent reports [9].

\section{Process Data}

For the patients receiving IV rt-PA, the average DTN time for administration of IV rt-PA decreased from year 1 to year 2 by approximately $20 \mathrm{~min}$ or $36 \%(\mathrm{p}=0.03$; table 3$)$. The DTI time decreased from year 1 to year 2 but was not significantly different. Procedure length only slightly decreased from year 1 to year $2(p=0.51)$. In both years, approximately three quarters of procedures were done with stentrievers and about $40 \%$ used IA medications (table 2). Three procedural complications were identified in year 1 and one in year 2 . In year 1 , there was one case of femoral artery dissection in an elderly patient with severe peripheral atherosclerotic disease. There were two intracerebral hemorrhages within $24 \mathrm{~h}$ of treatment and one case of ventilator-dependent respiratory failure requiring prolonged intubation and conversion to tracheostomy. Although the complication rate decreased from year 1 to year 2 , the decrease was not statistically significant.

\section{Discussion}

\section{CSC Requirements}

In 2000, the BAC established guidelines for a primary stroke center $[1,10]$, and in 2005, further requirements for a CSC were outlined [2]. Since 2005, many academic and community medical centers have sought to establish CSCs. Retrospective analysis has suggested that patient outcomes are improved when stroke care is organized into such centers [11-14]. For example, Kim et al. [15] calculated a $28 \%$ reduction in 30-day mortality after establishment of a CSC. Currently, there is a need to define expected progress during the initiation of a CSC and examine the benefits of EST. The aim of the present research is to address this need. 

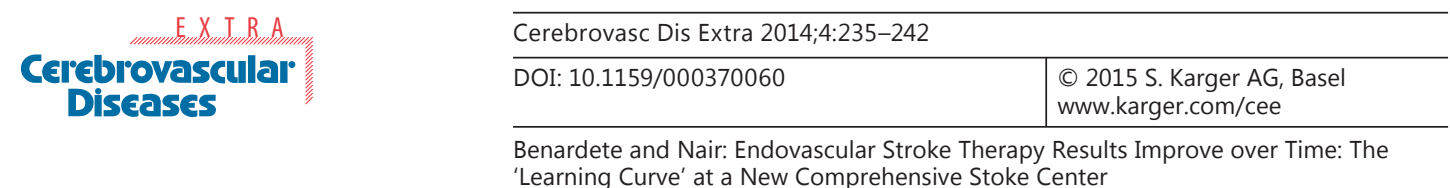

\section{Recent Randomized Clinical Trials}

Several recent clinical trials have purported to show the limited efficacy of EST for acute ischemic stroke [16-18]. These results created a great deal of discussion and re-evaluation of endovascular therapy protocols [19]. While some have suggested a moratorium of EST for stroke [20], finer analysis of the data suggests that certain patients may benefit from this therapy $[19,21,22]$. A number of authors have noted flaws in those trials including outdated treatment modalities, poor patient selection algorithms, and inadequate data analysis [23]. Our center has continued to offer EST because our selection criteria differ from those of the reported trials. We select patients based on two necessary criteria: (1) imaging evidence of significant ischemic penumbra and a vessel occlusion of CT angiography, and (2) ineligibility for IV rt-PA or failure to improve after $1 \mathrm{~h}$ of IV rt-PA. In addition, the prospective, randomized trials published in 2013 suggested that EST has a safety profile similar to IV rt-PA [16-18].

\section{Outcome Improvements}

At our CSC, we found that there was a statistically significant improvement in the number of independent patients at discharge ( $\mathrm{mRS} \leq 2)$ following EST in year 2 compared to year 1 . In addition, there were trends towards decreased mortality, decreased complications, and increased revascularization. Despite identical selection criteria, the patients in year 2 were slightly younger which may confound the results since our separate analysis has shown that older age is negatively correlated with a good outcome following EST. Others have shown a similar relationship between age and poor outcome [24, 25]. Nevertheless, our data suggests that improvement in a number of outcome metrics (e.g. TICI score, change in NIHSS score, and complication rates) also occurred from year 1 to year 2 , not all of which are likely due to the age variation in the patient population. Overall, for both years, approximately $40 \%$ of patients improved following EST. This result is similar to previous reports $[26,27]$.

\section{Process and Technical Improvements}

Our data suggests that there are improvements over time in the delivery of EST. In our series, there was a decrease in mortality and morbidity from year 1 to year 2 as well as an increase in excellent revascularization (TICI score $=3$ ), but these did not reach statistical significance. Although many individual improvements did not reach statistical significance, it is likely that the aggregate effect of these changes led to the statistically significant improvement in discharge status ( $\mathrm{mRS}$ score $\leq 2$ ).

We did observe statistically significant decreases in DTN time $(p=0.03)$ from year 1 to year 2. There were also trends towards decreased DTI time and procedure length from year 1 to year 2. In their study of stentriever use, Eesa et al. [28] have also shown procedural improvements over time. In our ANOVA analysis, increasing DTI time is associated with increased mortality suggesting that accelerating delivery of care in these complex systems is of value.

In summary, the initiation phase of a CSC is associated with significant improvements in outcome over time. These improvements may be attributed to increasing technical ability as well as to decreasing time in delivery of effective therapy. Furthermore, shorter time to intervention and successful revascularization are associated with improved outcomes.

\section{Acknowledgements}

The authors would like to thank Eileen Gallagher, RN, for her assistance with data collection and Robert H. Rosenwasser, MD, for his helpful suggestions and editorial advice. 


\section{Cerebrovascular \\ Diseases}

\section{Disclosure Statement}

The authors have no conflicts of interest to disclose.

\begin{tabular}{l|l}
\hline \multicolumn{2}{l}{ Cerebrovasc Dis Extra 2014;4:235-242 } \\
\hline DOI: $10.1159 / 000370060$ & $\begin{array}{l}\text { @ 2015 S. Karger AG, Basel } \\
\text { www.karger.com/cee }\end{array}$ \\
\hline
\end{tabular}

Benardete and Nair: Endovascular Stroke Therapy Results Improve over Time: The 'Learning Curve' at a New Comprehensive Stoke Center

\section{References}

1 Alberts MJ, et al: Recommendations for the establishment of primary stroke centers. Brain Attack Coalition. JAMA 2000;283:3102-3109.

-2 Alberts MJ, et al: Recommendations for comprehensive stroke centers: a consensus statement from the Brain Attack Coalition. Stroke 2005;36:1597-1616.

-3 Fugate JE, Klunder AM, Kallmes DF: What is meant by ‘TICI’? AJNR Am J Neuroradiol 2013;34:1792-1797.

4 Higashida R, et al: Trial design and reporting standards for intraarterial cerebral thrombolysis for acute ischemic stroke. J Vasc Interv Radiol 2003;14:S493-S494.

$\$ 5$ Tissue plasminogen activator for acute ischemic stroke. The National Institute of Neurological Disorders and Stroke rt-PA Stroke Study Group. N Engl J Med 1995;333:1581-1587.

6 Gory B, et al: Value of perfusion CT-guided recanalization therapy in acute ischemic stroke patients. Cerebrovasc Dis 2014;37:389-390.

7 Wintermark M, et al: Prognostic accuracy of cerebral blood flow measurement by perfusion computed tomography, at the time of emergency room admission, in acute stroke patients. Ann Neurol 2002;51:417-432.

$\$ 8$ Bruno A, et al: Improving modified Rankin Scale assessment with a simplified questionnaire. Stroke 2010;41: 1048-1050.

-9 Prabhakaran S, et al: Posttreatment variables improve outcome prediction after intra-arterial therapy for acute ischemic stroke. Cerebrovasc Dis 2014;37:356-363.

10 Mohammad YM, et al: Primary stroke center: basic components and recommendations. South Med J 2006;99: 749-752.

11 Gorelick PB: Primary and comprehensive stroke centers: history, value and certification criteria. J Stroke 2013;15:78-89.

12 Panezai S, et al: Compliance with joint commission measures in state-designated stroke centers. J Hosp Med 2014;9:88-93.

13 Berlet MH, et al: Does modern ischemic stroke therapy in a large community-based dedicated stroke center improve clinical outcomes? A two-year retrospective study. J Stroke Cerebrovasc Dis 2014;23:869-878.

14 Uehara T, et al: Which should be the essential components of stroke centers in Japan? A survey by questionnaires sent to the directors of facilities certified by the Japan stroke society. Cerebrovasc Dis 2014;37:409416.

15 Kim DH, et al: Organized comprehensive stroke center is associated with reduced mortality: analysis of consecutive patients in a single hospital. J Stroke 2013;15:57-63.

16 Broderick JP, et al: Endovascular therapy after intravenous t-PA versus t-PA alone for stroke. N Engl J Med 2013;368:893-903.

17 Ciccone A, et al: Endovascular treatment for acute ischemic stroke. N Engl J Med 2013;368:904-913.

18 Kidwell CS, et al: A trial of imaging selection and endovascular treatment for ischemic stroke. N Engl J Med 2013;368:914-923.

19 Singh B, et al: Endovascular therapy for acute ischemic stroke: a systematic review and meta-analysis. Mayo Clin Proc 2013;88:1056-1065.

20 Chimowitz MI: Endovascular treatment for acute ischemic stroke - still unproven. N Engl J Med 2013;368: 952-955.

21 Liebeskind DS: Trials of endovascular therapies or collaterals? Int J Stroke 2013;8:258-259.

22 Mokin M, et al: Endovascular treatment of acute ischemic stroke: the end or just the beginning? Neurosurg Focus 2014;36:E5.

23 Fargen KM, et al: Improvements in recanalization with modern stroke therapy: a review of prospective ischemic stroke trials during the last two decades. J Neurointerv Surg 2013;5:506-511.

24 Liggins JT, et al: A score based on age and DWI volume predicts poor outcome following endovascular treatment for acute ischemic stroke. Int J Stroke 2013, Epub ahead of print.

25 Mono ML, et al: Intra-arterial thrombolysis for acute ischemic stroke in octogenarians. Cerebrovasc Dis 2012; 33:116-122.

26 Burns TC, et al: Endovascular interventions following intravenous thrombolysis may improve survival and recovery in patients with acute ischemic stroke: a case-control study. AJNR Am J Neuroradiol 2008;29:19181924.

-27 Prabhakaran S, et al: Major neurologic improvement following endovascular recanalization therapy for acute ischemic stroke. Cerebrovasc Dis 2008;25:401-407.

28 Eesa M, et al: Mechanical thrombectomy with the Solitaire stent: is there a learning curve in achieving rapid recanalization times? J Neurointerv Surg 2014;6:649-651. 